\title{
El Diseño Urbano como experiencia docente: Estrategias y oportunidades de una formación temprana.
}

Urban design and teaching experience: Strategies and opportunities for early education.

Gonzalo Arze A. (garze@uchilefau.cl)

Profesor Asistente, Departamento de Urbanismo U. de Chile. Magíster en Urbanismo, U. de Chile

Constantino Mawromatis P. (cmawroma@uchile.cl)

Profesor Asistente, Departamento de Urbanismo U. de Chile. DEA, UPM.

Felipe Corvalán T. (felipecorvalan@uchilefau.cl)

Instructor, Departamento de Arquitectura U. de Chile. Magíster en Arte con mención en Teoría e Historia, U. de Chile

Hernán Orozco R.

Arquitecto, U. de Chile. Diplomado en Planificación Urbana Integral, P. U. Católica de Chile.

\section{Filiación}

Facultad de Arquitectura y Urbanismo, Universidad de Chile

\section{Resumen}

El presente artículo recoge la experiencia de elaboración y puesta en funcionamiento de un Taller de Diseño Urbano en el contexto del tercer año de la Carrera de Arquitectura de la Facultad de Arquitectura y Urbanismo de la Universidad de Chile. Esta experiencia docente permite al estudiante aproximarse a las problemáticas más relevantes de la ciudad contemporánea, así como la posibilidad de reflexionar en torno a las estrategias formativas bajo las cuales se desarrolla el futuro arquitecto. El paso desde una ejercitación proyectual centrada en la definición y diseño del objeto arquitectónico hacia la comprensión de la ciudad como campo de acción integral de las intervenciones arquitectónicas, es propuesto como una acción relevante tendiente a sensibilizar al estudiante con la ciudad y el Diseño Urbano.

\section{Palabras Claves}

Taller; Diseño Urbano; Enseñanza de la Arquitectura.

\begin{abstract}
This paper describes and analyzes the process of design and implementation of an Urban Design Studio for third year architecture students at the Faculty of Architecture and Urbanism of the University of Chile. This academic experience has allowed the faculty to pass on to the students all the necessary tools to approach the most relevant issues of the contemporary city, as well as the opportunity to debate about the pedagogical strategies under which future architects are being trained.

The aim of this academic experience is to shift the focus of design exercises, from the development of architectural objects to a more profound understanding of the urban realm as the main field of action of every architectural intervention, in order to make students more aware about the city and Urban Design.
\end{abstract}

\section{Key Words}

Design studio; Urban Design; Architectural Education. 
REVI STA DE

URBANI SMO

ISSN 0717-5051

http://revistas.uchile.cl/index.php/RU/index
El Diseño Urbano como experiencia docente: Estrategias y oportunidades de una formación temprana. Urban design and teaching experience: Strategies and opportunities for early education.

\section{Sumario}

Introducción.

Marco teórico: La ciudad contemporánea entre la crisis y la oportunidad.

Metodología: Experiencia docente en el Diseño Urbano desde una formación temprana.

Resultados y conclusiones: El taller, sus dificultades, los logros y los desafíos.

Referencias Bibliográficas

\section{I ntroducción}

La acción docente universitaria en relación al diseño urbano, en la carrera de arquitectura, constituye una oportunidad para analizar las distintas variables que concurren en el diagnóstico de los conflictos presentes en las ciudades contemporáneas, desde una perspectiva crítica y desprejuiciada. Con ello se insta al debate y la reflexión orientada a la construcción de un discurso propio y coherente por parte del estudiante, basado en principios que operen a partir del bien común. Nacen por tanto, y de forma natural, las inquietudes por poder derivar dichos principios en planteamientos que se sostengan en una visión alternativa, contestataria y a la vez proactiva respecto a los ámbitos que inciden en la habitabilidad de la ciudad.

La aproximación al Diseño Urbano desde una perspectiva académica permite identificar las oportunidades que ofrece el aprender de la historia de nuestros asentamientos urbanos, el explorar e innovar a partir de una actitud de permanente cuestionamiento, desprendida de las dificultades y aparente inexorabilidad del llamado mundo real, asociado a la debilidad de los marcos normativos de la planificación, la precariedad institucional y el bajo compromiso político con el desarrollo y gestión de una visión integral de Diseño Urbano, tal como ocurre en el plano nacional con por ejemplo, la carencia de instrumentos locales que incorporen aspectos tridimensionales en sus normativas. De las universidades, fundamentalmente de las que tienen un compromiso público, pueden emanar las respuestas que den cuenta de un cambio de paradigma, que permitan pasar desde un desarrollismo cuantitativo hacia una concepción cualitativa y sustentable, articulando una visión holística del Diseño Urbano en el contexto contemporáneo.

La posibilidad de desarrollar un Taller de Diseño Urbano en los niveles intermedios de la Carrera de Arquitectura, permite relevar la formación del futuro profesional en los ámbitos de la escala y complejidad de la ciudad, desarrollando una mirada integral, teniendo siempre al ciudadano como sujeto fundamental en la concepción de planteamientos y propuestas creativas, inclusivas y responsables con el medio ambiente natural y construido, valorizando el patrimonio tangible e intangible.

La actividad proyectual propia de la asignatura del Taller, permite dar luces sobre el rol del Diseño Urbano en el ámbito académico y también profesional, ya sea como ampliación y complemento de la práctica tradicional del Diseño Arquitectónico o bien, como la manifestación de la evolución de la enseñanza tradicional de la disciplina del urbanismo hacia la generación de nuevas estrategias de entendimiento y planificación del territorio. A modo de diagnóstico, es posible evidenciar una tendencia formativa consolidada en los talleres de pregrado que privilegia el ejercicio de diseño centrado en la producción del objeto arquitectónico. Frente a esta condición, reconociendo que la arquitectura ineludiblemente requiere de la definición compositiva, material y espacial del edificio, la propuesta de un Taller de Diseño Urbano plantea la necesidad de ampliar esta comprensión, sensibilizándose con las demandas mayores 

oportunidades de una formación temprana. Urban design and teaching experience: Strategies and opportunities for early education.

que exige la ciudad. Bajo estos términos, las interrogantes que movilizan la propuesta del Taller son ¿cómo desarrollar una visión crítica en el estudiante frente a los modelos de desarrollo urbano vigentes y en proceso de implementación?, ¿cómo fomentar la comprensión de las respuestas arquitectónicas particulares asociadas a un sistema de relación mayor que determina su presencia, función y morfología? y finalmente, ¿qué competencias estimular en los estudiantes para que puedan intervenir responsable y reflexivamente sobre la ciudad? De esta manera el objetivo principal del Taller es permitir que los alumnos puedan construir una mirada crítica y desprejuiciada sobre la ciudad, para de esta manera poder intervenir responsablemente sobre ella.

\section{Marco teórico: La ciudad contemporánea entre la crisis y la oportunidad.}

La aproximación al concepto de proyecto permite establecer los límites dentro de los cuales tradicionalmente se ha desarrollado la enseñanza y la formación del arquitecto. Un concepto que entendido modernamente podrá ser pensado como el encuentro entre teoría y práctica, tal como es sugerido por Leon Battista Alberti en el tratado De re aedificatoria, escrito en el siglo $\mathrm{XV} .{ }^{1}$ La mirada de Alberti, pese al tiempo transcurrido y a las transformaciones sucedidas al interior del campo arquitectónico, nos propone entender a la arquitectura como un ejercicio intelectual que supera las barreras del mero hacer y de la evidencia tangible de su presencia, siempre en concordancia con las necesidades de sus habitantes. Una disciplina que precisamente, a partir de su condición teórica - práctica, puede ser asociada con un espacio formativo basado en la permanente interacción entre la enseñanza y el aprendizaje, entre lo propuesto por los equipos docentes y la dinámica participación de los estudiantes. ${ }^{2}$

A partir de lo anteriormente señalado, resulta necesario reflexionar en torno a la formación del arquitecto en el contexto de la sociedad contemporánea. Un mundo que se complejiza a la par de su continuo desarrollo y en el que se hace evidente la crisis de aquéllos modelos de enseñanza consolidados en el siglo pasado. Un ámbito de acción que requiere por parte del futuro arquitecto el desarrollo de la capacidad de adaptación frente a un escenario en permanente transformación.

La evidencia abunda en cuanto a los diversos conflictos presentes en la ciudad contemporánea, los cuales inciden en el progresivo deterioro de la calidad de vida de sus habitantes. Ello se ve agravado por la tendencia de crecimiento de la población urbana a nivel global, producto de las ventajas comparativas que ofrecen especialmente los grandes núcleos urbanos. Las proyecciones de crecimiento de la población urbana a nivel global en las próximas décadas configuran un cuadro de gran complejidad en el desarrollo de las ciudades, tanto en el presente como en el futuro. ${ }^{3}$ Un contexto en el que no sólo influyen las demandas disciplinares, sino también las de una ciudadanía cada vez más empoderada.

\footnotetext{
${ }^{1}$ Al respecto Leon Battista Alberti establece: "Yo por mi parte, voy a convenir que el arquitecto será aquel que con un método y un procedimiento determinados y dignos de admiración haya estudiado el modo de proyectar en teoría y también de llevar a cabo en la práctica cualquier obra que, a partir del desplazamiento de los pesos y la unión y ensamble de los cuerpos, se adecue, de una forma hermosísima a las necesidades propias de los seres humano". ALBERTI, L. Battista. (1991).De Re Aedificatoria. Prólogo de Javier Rivera; traducción de Javier Fresnillo Núñez. Madrid, España: Edición Akal. Pág. 51. Originalmente publicado el año 1452.

2 Tal como es establecido por Jean Francois Mabardi "Esta definición nos enfrenta a una dificultad: la arquitectura está en el medio del río, entre enseñanza y aprendizaje." Una situación propone un escenario formativo que pretende estimular la participación activa por parte de los estudiantes, activando su capacidad exploratoria. MABARDI, J.F. (2012) . Maestría el proyecto. Apuntes para la práctica de la enseñanza del proyecto. Ediciones Universidad del Bío-Bío. Pág. 76.

3 Según el informe de las Naciones Unidas 2010 Revision of the World Population Prospects, la población mundial es cercana a los 7 mil millones en la actualidad, proyectándose un estimado de 10,1 mil millones de habitantes para el 
REVISTA DE

URBANI SMO

ISSN 0717-5051

http://revistas. uchile.cl/index.php/RU/index
El Diseño Urbano como experiencia docente: Estrategias y oportunidades de una formación temprana. Urban design and teaching experience: Strategies and opportunities for early education.

Dicho crecimiento se canaliza en gran medida a través del modelo de ciudad dispersa y difusa cuestionada hace décadas en su propio lugar de origen-, representando hoy uno de los principales motores de la economía de muchas regiones a nivel global, concentrándose tanto en las grandes ciudades como en centros urbanos emergentes y ciudades intermedias, abarcando diversos contextos geográficos y culturales. Las tasas de crecimiento de las superficies involucradas superan largamente los aumentos de la población correspondiente; vale decir, el crecimiento del ámbito suburbano se incrementa exponencialmente y con ello la dependencia del automóvil agudiza las transformaciones sobre el territorio y las formas de vida de la gente (Ward Richardson y Chang-Hee, 2004) / (Urban sprawl in Europe, 2006. Informe no 10/2006 de la Agencia Europea de Medio Ambiente), anexando predios rurales a los límites urbanos en una continua sumatoria de paños monofuncionales de bajas densidades asociados a las autopistas y los nuevos artefactos urbanos que dominan hoy en día el paisaje suburbano. En efecto, el crecimiento desregulado por extensión desencadena una sucesión de impactos negativos sobre la organización del territorio y la calidad de vida, afectando la movilidad cotidiana, el encuentro ciudadano, el sentido de pertenencia y apropiación generadora de identidad, entre muchas otras consecuencias asociadas a la pobreza morfológica que caracteriza a los megaproyectos residenciales de la periferia urbana. Más que una consecuencia del laissez faire, esto es producto de decisiones concientes funcionales a la especulación inmobiliaria (Duany, 1991), situadas al margen de las decisiones que emergen desde el interés público y en beneficio de toda la sociedad.

Así mismo, el desarrollo de los centros históricos y posteriores ensanches o expansiones de ciudades tradicionales o núcleos emergentes no logran igualar el dinamismo de las periferias y de la interfaz urbano-rural. Si bien abundan notables experiencias de revitalización de áreas deprimidas en los centros y pericentros urbanos, especialmente en el caso europeo, prevalece por un lado la tendencia hacia el deterioro de dichas áreas, y por otro lado la regeneración urbana a partir de políticas de densificación sin un proyecto urbano, permitiendo e incentivando la gentrificación y la respectiva consecuencia de exclusión, la destrucción del patrimonio tangible e intangible y la pérdida de identidad del lugar, desdibujando el tejido urbano y social. Es el caso recurrente de políticas de fomento a la construcción en altura con escaso compromiso hacia el espacio público, ya sea a través de incentivos al sector privado o, a partir de programas públicos que intervienen el espacio urbano, que en definitiva no dan cabida a la impronta del Diseño Urbano. Generalmente, el progresivo abandono y subsecuente deterioro de estas áreas centrales de la ciudad se asocian posteriormente a procesos especulativos que distorsionan u orientan la inversión privada de acuerdo a visiones cortoplacistas, desprovistas naturalmente de las lógicas de la planificación en función del bien común.

No obstante los conflictos presentes en los distintos ámbitos del funcionamiento y quehacer urbano, surgen oportunidades que permiten reconocer las potencialidades de la vida urbana, encausando modalidades alternativas de orientar el desarrollo, compatibilizando el dinamismo de la actividad inmobiliaria con las necesidades y los anhelos de una población más activa y conciente de su medio ambiente construido, una ciudadanía agobiada por los problemas que provienen de la prolongada desatención por la urbe y ávida de encontrar modelos que puedan

año 2100, y 9,3 para mediados del presente siglo, basándose en las actuales proyecciones de fertilidad. En cuanto a las proyecciones de aumento de la población urbana mundial, según el United Nations Population Division, ésta se incrementaría desde los 3.494.607 millones en el año 2010, a 6.398.291 millones en el 2050, reflejando un explosivo aumento de la población urbana a nivel global. En cuanto a la población rural mundial, ésta se reduciría considerablemente de los 3.411 .951 millones en 2010, a 2.792.995 millones de habitantes en el año 2050.

5 Departamento de Urbanismo - FAU - Universidad de Chile 
proporcionar mejores condiciones de vida. Esperanzadoras son las improntas que señalan otras formas de concebir el desarrollo, igualmente dinámico pero más sustentable, con atención hacia las variables humanas y estéticas de la ciudad, incorporando nuevas metodologías y herramientas con las cuales el Diseño Urbano está incidiendo en el medio. En tal dirección cobran cada vez más notoriedad las tendencias de "crecimiento inteligente" (smart growth) que surgen de los principios del Nuevo Urbanismo norteamericano; las aproximaciones ecológicas del diseño urbano integrando los intereses y requerimientos de la gente, las comunidades y el medio ambiente, con propuestas innovadoras enfocadas a la sostenibilidad en el tiempo; y las concepciones de una movilidad sostenible, que orienten los nuevos proyectos urbanos.

Entender estas nuevas dinámicas y propiciar su diálogo con la realidad local parece ser una tarea inconclusa y necesaria, permitiendo la reflexión en torno a las políticas que orientan y organizan el desarrollo de las ciudades en Chile. En este contexto la acción docente en el ámbito del diseño urbano se comprende como fundamental para orientar e incentivar tempranamente a las futuras generaciones de profesionales desde la convicción ética y estética de aportar desde el discurso o la acción proyectual a la construcción y logro de mejores espacios públicos, mejores barrios y ciudades en nuestro país.

\section{Metodología: Experiencia docente en el Diseño Urbano desde una formación temprana.}

Frente a la relevancia y responsabilidad de instar y orientar a los alumnos de la Carrera de Arquitectura en cuanto a formular sus propios discursos relativos a la ciudad como espacio integrador y democratizador surge la posibilidad de incorporar el Diseño Urbano en la malla curricular desde las instancias de un Taller de Arquitectura. Es también una oportunidad para entender la disciplina del Diseño Urbano desde los marcos epistemológicos de la arquitectura y el urbanismo y asimismo, desde diversos ámbitos de aproximación, ya sea desde la movilidad, la habitabilidad, la integración, etc., vinculando las distintas escalas y complejidades; por otro lado es también la instancia para velar por la congruencia entre la identificación de los problemas, su debido diagnóstico y la acción proyectual fundamentada.

Es así como en año 2012 se ofrece por primera vez un Taller de Diseño Urbano dentro de la malla de los talleres de tercer año de la Carrera de Arquitectura, asignatura identificada oficialmente como "Taller de Diseño Arquitectónico 1 y $2^{\prime \prime}$ correspondiente al $5^{\circ}$ y $6^{\circ}$ semestre académico de la Facultad de Arquitectura y Urbanismo de la Universidad de Chile. Surge declarando la voluntad de fortalecer, desde una etapa temprana, el aspecto crítico y proyectual en la formación del estudiante de arquitectura en lo relativo a la escala y complejidad urbana en consideración a:

- La identidad, tradición y vocación para con el Urbanismo que ha marcado a la Facultad desde su nombre institucional y su sello formativo, lo cual distingue a los arquitectos de la Universidad de Chile respecto a otras escuelas de arquitectura, constituyendo por tanto, un atributo y fortaleza que debe preservarse y potenciarse a raiz de los desafíos actuales y futuros del hábitat contemporáneo.

- Los desafíos y diversificación que sugiere el ejercicio profesional actual y futuro, adquiriendo cada vez mayor urgencia lo referido a temas relacionados con la habitabilidad y sostenibilidad de las ciudades, lo cual debe enfrentarse crecientemente desde la disciplina del diseño urbano a partir de una concepción holística de la disciplina.

- El perfilamiento por parte del estudiante, hacia temáticas relativas al Diseño Urbano en los niveles intermedios, facilitando y potenciando la educación continua hacia futuros programas de posgrado en las líneas disciplinares afines. 
REVISTA DE

URBANI SMO

ISSN 0717-5051

http://revistas.uchile.cl/index.php/RU/index
El Diseño Urbano como experiencia docente: Estrategias y oportunidades de una formación temprana. Urban design and teaching experience: Strategies and opportunities for early education.

En tal sentido, la generación de un Taller que permita al estudiante de pregrado vincular el Diseño Arquitectónico con el Diseño Urbano, resulta una oportunidad para repensar las estrategias de enseñanza de la arquitectura y la vinculación de éstas con el medio. De esta manera, el Diseño Urbano es entendido como aquel ámbito de acción proyectual que permite vincular a la arquitectura con la ciudad, un ejercicio que no sólo supone la debida contextualización de las intervenciones habituales provenientes desde la arquitectura, sino más bien, la apropiación de la ciudad como ámbito de experimentación urbano-arquitectónica.

Esta aproximación intenta establecer una mirada integral sobre la relación que se produce entre la arquitectura como respuesta específica y la ciudad como campo de interacción e integración de tales respuestas. Con esta orientación, el taller evita la comprensión del Diseño Arquitectónico centrado exclusivamente en la producción objetual, sumando a la etapa habitual de propuesta, instancias que permitan al alumno analizar y problematizar a partir del contexto de acción en que se insertaran sus intervenciones.

Paralelamente a lo anteriormente señalado, el Taller se plantea, asumiendo los desafíos que emanan de la modernización curricular en estudio para la Carrera de Arquitectura de la Universidad de Chile, como una instancia concreta de vinculación entre las líneas teóricas y la ejercitación o acción proyectual, dotando de las herramientas necesarias al alumno en cuanto a las habilidades reflexivas y críticas, y las capacidades para detectar y diagnosticar las problemáticas, ampliando el perfil tradicional del arquitecto hacia un profesional que sustente sus planteamientos a partir de un cuerpo teórico y técnico coherente e innovador. Para ello se contempla el integrar conceptos y aproximaciones contemporáneas de la disciplina, fomentando la reflexión, la participación y el debate, con el objeto de enriquecer la experiencia del taller como parte del proceso formativo de la carrera de arquitectura.

Esta experiencia es propuesta como una instancia clave, en la medida en que se compromete con la generación de futuros profesionales reflexivos y sensibles con el entorno urbano y el territorio en el que se insertan sus propuestas. Una visión que se enfrentan al crecimiento urbano fragmentario, excluyente y de escasa conectividad, propio de la ciudad dispersa, así como también a la especulación inmobiliaria que redefine el tejido urbano y social previamente consolidado al interior de las ciudades; una realidad que repercute invariablemente en la configuración territorial y en la forma en que se desarrolla la ciudad.

Junto con proponer problemáticas específicas vinculadas a la ciudad, el taller busca estimular y desarrollar de manera creciente el actuar autónomo por parte del alumno. Así, si bien es el equipo docente quien plantea las temáticas que serán abordadas por el taller, se espera que el estudiante sea capaz de reconocer problemáticas y posibilidades de intervención al interior de la ciudad, cuestión que le permitirá significar su propio trabajo y en ocasiones ampliar los límites de las temáticas inicialmente planteadas. De esta manera, al mismo tiempo que se exponen las tendencias discursivas y operacionales que hoy rigen la práctica del Diseño Urbano, el Taller promueve que el alumno pueda desarrollar un discurso propio, que le permita orientar y solventar las decisiones tomadas, formalizadas en la ciudad a través del ejercicio proyectual.

En términos metodológicos, la aproximación al Diseño Urbano propuesta por el taller intenta vincular de manera interactiva estrategias analíticas y estrategias operacionales, en un marco de coherencia y también de innovación, buscando que las intervenciones urbanoarquitectónicas respondan a una problemática detectada y debidamente analizada. Bajo estos parámetros, se definen tres etapas proyectuales básicas: etapa analítica, etapa de diagnóstico y problematización, y finalmente un etapa propositiva. De esta manera, es posible evidenciar un proceso continuo de materialización, a través de propuestas concretas de diseño, de las 


\section{REVISTA DE}

\section{URBANI SMO}

ISSN 0717-5051

http://revistas.uchile.cl/index.php/RU/index
El Diseño Urbano como experiencia docente: Estrategias y oportunidades de una formación temprana. Urban design and teaching experience: Strategies and opportunities for early education.

distintas concepciones e ideas generadas por los estudiantes. Del mismo modo, tal proceso implica el paso desde un inicial trabajo colaborativo, en el cual los alumnos del taller se organizan en grupos, hasta el desarrollo individual de alguno de los aspectos especialmente relevantes definidos en las propuestas generales de la etapa grupal inicial.

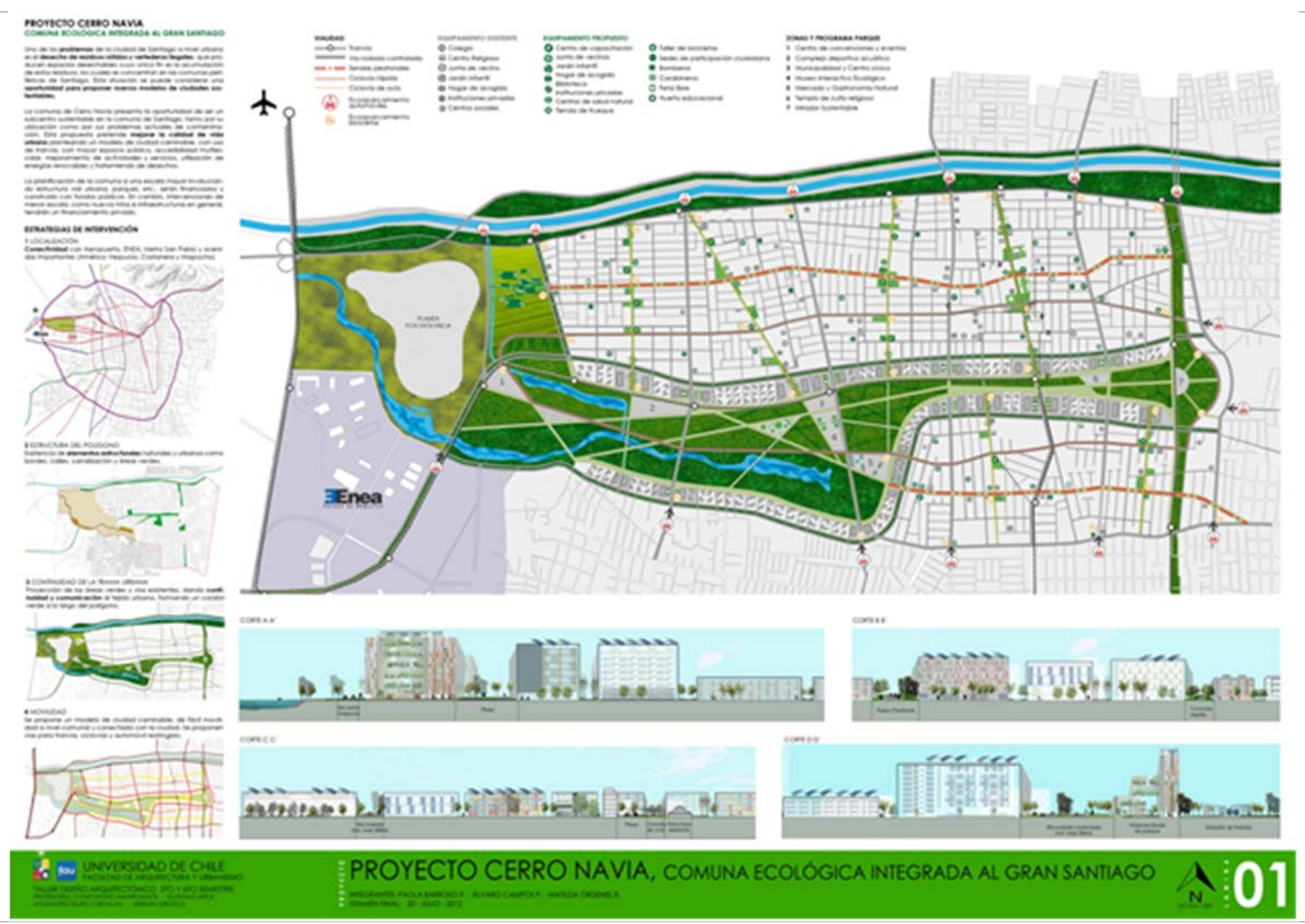

Fig 1. Propuesta Comuna Ecológica integrada al Gran Santiago. Fte.: Paola Barroso, Álvaro Campos, Matilda Órdenes

En este escenario, se busca que el estudiante sea capaz de confrontar ideas, conformar equipos de trabajo y construir su postura individual frente a las problemáticas abordadas, siempre en concordancia con una concepción global de la ciudad, asumiendo que la parte debe saber integrarse y dialogar con el todo, vinculando lo particular con lo complejo. Una instancia que pretende fomentar y promover la generación de propuestas innovadoras y alternativas por parte de los estudiantes que permitan redefinir el crecimiento y desarrollo urbano a partir de modelos sostenibles en el tiempo, que incorporen variables cualitativas y que se comprometan con el mejoramiento de las condiciones de habitabilidad de sus ciudadanos. En definitiva, un intento por motivar a los estudiantes a desarrollar una actitud proactiva y atenta frente al contexto construido en que se desenvuelven, que les permita construir un discurso propio orientado a la generación y planteamiento de un proyecto de ciudad y que a la vez, pueda ser sustentado consecuentemente de acuerdo a las variables analizadas y los recursos disponibles. 


\section{REVISTA DE}

\section{URBANI SMO}

ISSN 0717-5051

http://revistas. uchile.cl/index.php/RU/index
El Diseño Urbano como experiencia docente: Estrategias y oportunidades de una formación temprana. Urban design and teaching experience: Strategies and opportunities for early education.
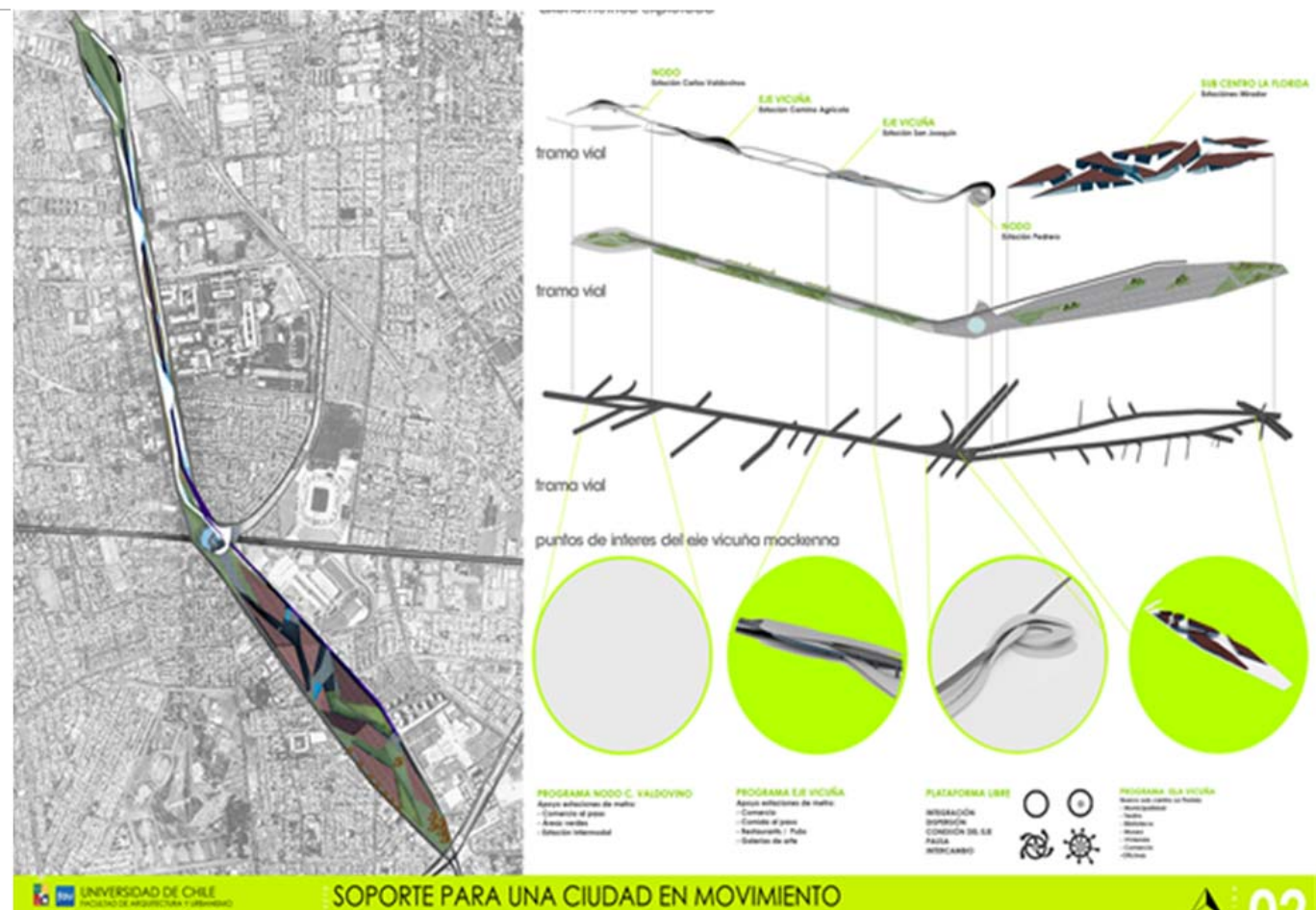

tromo vid
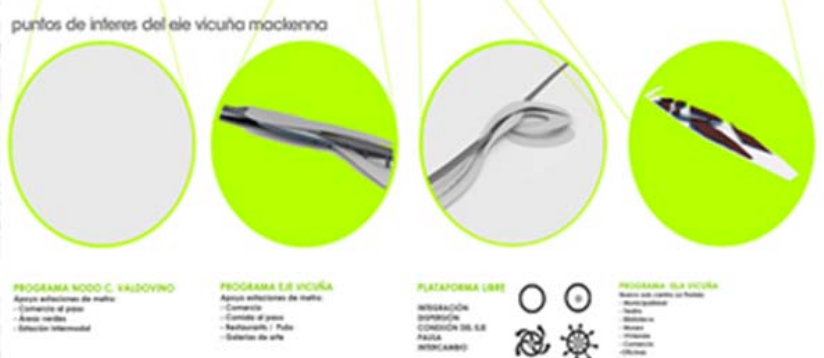

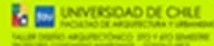

SOPORTE PARA UNA CIUDAD EN MOVIMIENTO

$\equiv$

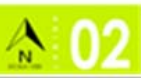

Fig 2. Propuesta de intervención sobre Eje Vicuña-Mackena. Fte.: Diego Díaz, Daniel Espinoza, Florencia Moure.
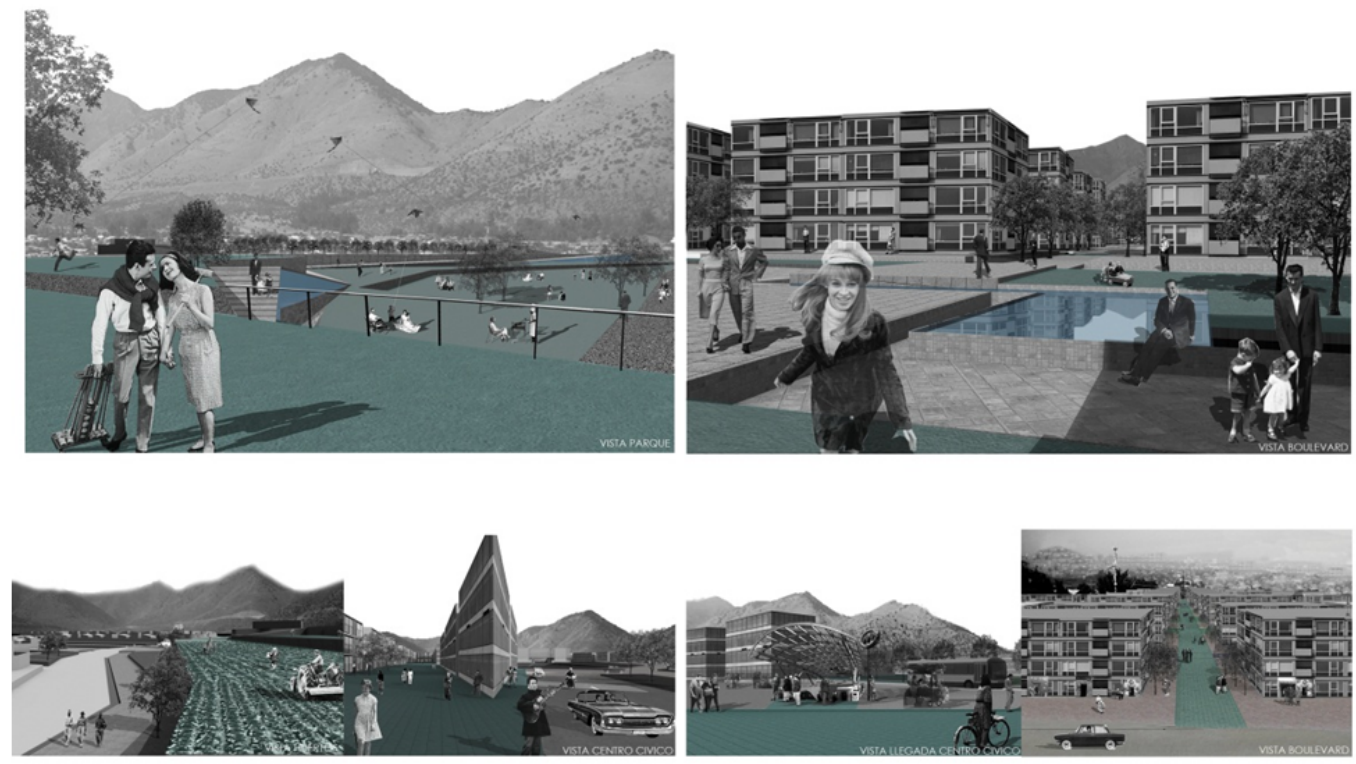

Ii. Fou UNIVESSIDAD DE CHILE

RENOVACION URBANA LA PINCOYA

命 02

Fig 3. Propuesta de renovación urbana Población la Pincoya. Fte.: Nicolás Benoit, Cesar Carrasco, Francisco Espinoza. 9 


\section{Resultados y conclusiones: El taller, sus dificultades, los logros y los desafíos.}

La oportunidad de proponer un Taller de Diseño Urbano en el proceso de formación del futuro arquitecto, nos enfrentó al desafío de estimular y desarrollar en los alumnos la capacidad de identificar y analizar de manera crítica y responsable las problemáticas asociadas a la configuración de la ciudad y las variables que en ésta inciden. Paralelamente, permitió dar paso a la generación de acciones concretas de intervención por parte de los estudiantes, resolviendo las carencias detectadas o bien imaginando y descubriendo de manera reflexiva las oportunidades y potencialidades aun no exploradas de las áreas estudiadas.

En tal dirección, una de las premisas iniciales asumidas por el Taller fue la necesidad de repensar las condiciones en las cuales se entiende y produce el vínculo entre objeto arquitectónico y contexto urbano. Una primera mirada que apunta a la comprensión del diseño urbano como la sumatoria e integración de estrategias analíticas y operacionales que actúan sobre la ciudad y el territorio, abordando de manera integral las problemáticas de los asentamientos urbanos (Munizaga, 1992). Una segunda aproximación intentó evidenciar el juego de relaciones espaciales que se produce entre los elementos volumétricos, edificios $u$ otros, y los espacios exteriores que delimitan y, cuestión que constituye la esencia del objeto del Diseño Urbano (López, 1999). De esta manera, las tres etapas principales que articularon el trabajo de los alumnos a lo largo del taller, Análisis - Problematización-Propuesta de Diseño, fueron desarrollados bajo esta exigencia de integración e inclusividad, asociada a un diálogo permanente entre el todo y las partes, entre las propuestas específicas de diseño y su inserción en el contexto urbano mayor que configura a la ciudad. Una cuestión que resulta fundamental si aceptamos que la tendencia y orientación de la formación del estudiante de arquitectura, sobre todo en los primeros años de la carrera, se centra en la ejercitación del diseño asociado a respuestas específicas, claramente acotadas, que permitan la posterior materialización del objeto arquitectónico.

Las distintas propuestas desarrolladas en el Taller se emplazaron en la Región Metropolitana y permitieron identificar por parte de los estudiantes, la diversidad de problemáticas y oportunidades asociadas a la ciudad de Santiago, propiciando nuevas lecturas de sectores tradicionales o bien, descubriendo aspectos desconocidos de la ciudad. En términos de resultados inicialmente obtenidos por el taller, podemos establecer que la experiencia hasta aquí desarrollada ha permitido que los alumnos resinifiquen su vínculo con la ciudad. La metodología utilizada (análisis/problematización/propuesta) ha dado como resultado la convivencia en el taller de distintas áreas y temas de interés identificados por los estudiantes, tal como ejemplifican las imágenes que acompañan este artículo. La sustentabilidad materializada en la propuesta de un Eco Barrio, el transporte y la movilidad como un tema esencial de la configuración de la ciudad, la renovación urbana como estrategia de integración social o la imagen urbana como herramienta esencial del Diseño Urbano, son algunas de las temáticas que se han abordado en el taller, convirtiendo a cada una de las sesiones en un punto de confluencia diversos, pero al mismo tiempo complementario, que exige a los alumnos una comprensión integral de la ciudad. Un conjunto de aproximaciones que configuraron un Taller marcado por la diversidad y complementariedad del análisis y de las temáticas de estudio, permitiendo un debate profundo en torno a los aspectos más representativos que configuran a la ciudad contemporánea.

En cuanto a la identificación de las principales dificultades asociadas a la incorporación y desarrollo de este Taller de Diseño Urbano, bien podríamos recurrir y hacer referencia a la idea de escala. Un concepto que si bien está directamente asociado a cuestiones físico-territoriales y a las estrategias de representación, también nos permite reflexionar en torno al desarrollo de 

oportunidades de una formación temprana. Urban design and teaching experience: Strategies and opportunities for early education.

las capacidades analíticas en los estudiantes, comprendiendo a la ciudad de manera integral, estableciendo asociaciones entre las distintas variables que configuran un determinado problema urbano-arquitectónico. La dificultad de pasar desde soluciones abordadas a partir de una escala de resolución 1:100 o 1:50, propia de los talleres de diseño arquitectónico, a proyectos que superan ampliamente tales parámetros de intervención, constituyó un obstáculo permanente en el trabajo de los alumnos a lo largo de todo el semestre. Las dudas permanente relativa a los límites de las distintas propuestas a desarrollar, evidenciada en interrogantes tales como ¿hasta dónde intervenir?, ¿cuáles son los límites del área de trabajo?, sólo pudieron ser resueltas en la medida en que los alumnos lograron entender su propuestas de manera integral y sistémica, identificando y descartando variables asociadas a sus propuestas de diseño, definiendo los limites analíticos, operativos y físicos de las intervenciones. En otras palabras, la problemática y limitaciones derivadas del traspaso desde la escala del objeto a la escala de la ciudad, es también homologable a un desarrollo en la escala de pensamiento que espera del estudiante y del cuerpo académico que lo guía, un pensamiento holístico, modificando y ampliando las estrategias de aproximación al diseño urbano-arquitectónico.

Asimismo, en la búsqueda de la fundamentación teórica del proyecto de Diseño Urbano, se establece como una dificultad la tendencia a establecer conceptos teóricos o abstractos como formas o elementos que configuran físicamente un sistema urbano, es decir, la asociación directa de conceptos como sustentabilidad, patrimonio o participación ciudadana como herramientas de diseño, sin entender la profundidad de la discusión propia de cada una de las temáticas o las diferencias entre los distintos modelos discursivos asociados a estos conceptos. En este contexto, se vuelve una tarea del equipo docente explicar estos modelos discursivos de planificación, gestión o crecimiento de las ciudades, para que el estudiante pueda traducirlos a "estrategias de diseño", es decir, herramientas técnico-materiales como pueden ser la eficiencia energética de la forma urbana, compacidad de la ciudad, procesos ecológicos aplicados al diseño urbano, etc. Esta misma dificultad se encuentra en los alcances teóricos correspondientes a la viabilidad, rentabilidad económica y social, o gestión de los proyectos urbanos.

Así, tal como fue establecido por Alberti hace más de quinientos años, el taller intenta evidenciar el necesario vínculo entre teoría y práctica como base del ejercicio proyectual. Una vinculación que tal como establece el arquitecto norteamericano Stan Allen necesita ser repensado en el contexto contemporáneo, entendiendo a las propuestas arquitectónicas y urbanas ya no como mandatos o imposiciones sobre el espacio, sino más bien como intervenciones que inevitablemente deberán interactuar con la realidad, sensibilizándose frente a sus requerimientos, pasando, tal como el propio Allen propone, desde una concepción objetual a una concepción de campo, que entienda a la arquitectura como una disciplina de circunstancia, en permanente iteración con la ciudad y sus habitantes. Precisamente la referencia al concepto de campo tal como es desarrollado por Allen ${ }^{4}$ permite sintetizar la orientación sugerida por el taller, basada en el entendimiento de la arquitectura como una disciplina permitir el diálogo de sus preceptos teóricos con el conjunto de variables y afección

\footnotetext{
4 “El término 'condición de campo' es al mismo tiempo una reafirmación de los cometidos de la arquitectura contextual y una propuesta para poder cumplir su programa. Las condiciones de campo se mueven de la unidad a la multiplicidad, de individuos a colectivos, de objetos a campos. Los arquitectos no sólo deben trabajar en oficina o estudio (en el laboratorio), sino también en el campo: en el sitio, en contacto con la fábrica de arquitectura. 'Sondeo de campo', 'oficina de campo', 'verificar en campo', 'condiciones de campo', implica la aceptación de lo real con toda su confusión e imprevisibilidad. Abre la arquitectura a la improvisación en el lugar. Las condiciones de campo consideran las restricciones como oportunidad y van más allá de la ética modernista-y estéticas-de transgresión. Al trabajar con y no contra el sitio provoca algo nuevo al registrar la complejidad de lo dado". (Allen, 1997, p.24)
}

11 


\section{REVISTA DE}

\section{URBANI SMO}

ISSN 0717-5051

http://revistas.uchile.cl/index.php/RU/index
El Diseño Urbano como experiencia docente: Estrategias y oportunidades de una formación temprana. Urban design and teaching experience: Strategies and opportunities for early education.

que definen las practicas cotidianas al interior de la ciudad, ampliando no sólo las escalas territoriales sino también y sobre todo, las escalas de pensamiento. Una exploración abierta, que requiere del trabajo colaborativo entre los propios estudiantes, que les permita entender que cada una de las decisiones tomadas y acciones implementadas incidirá inevitablemente en su contexto de inserción, superando los limites aparentemente acotados que contiene la forma arquitectónica.
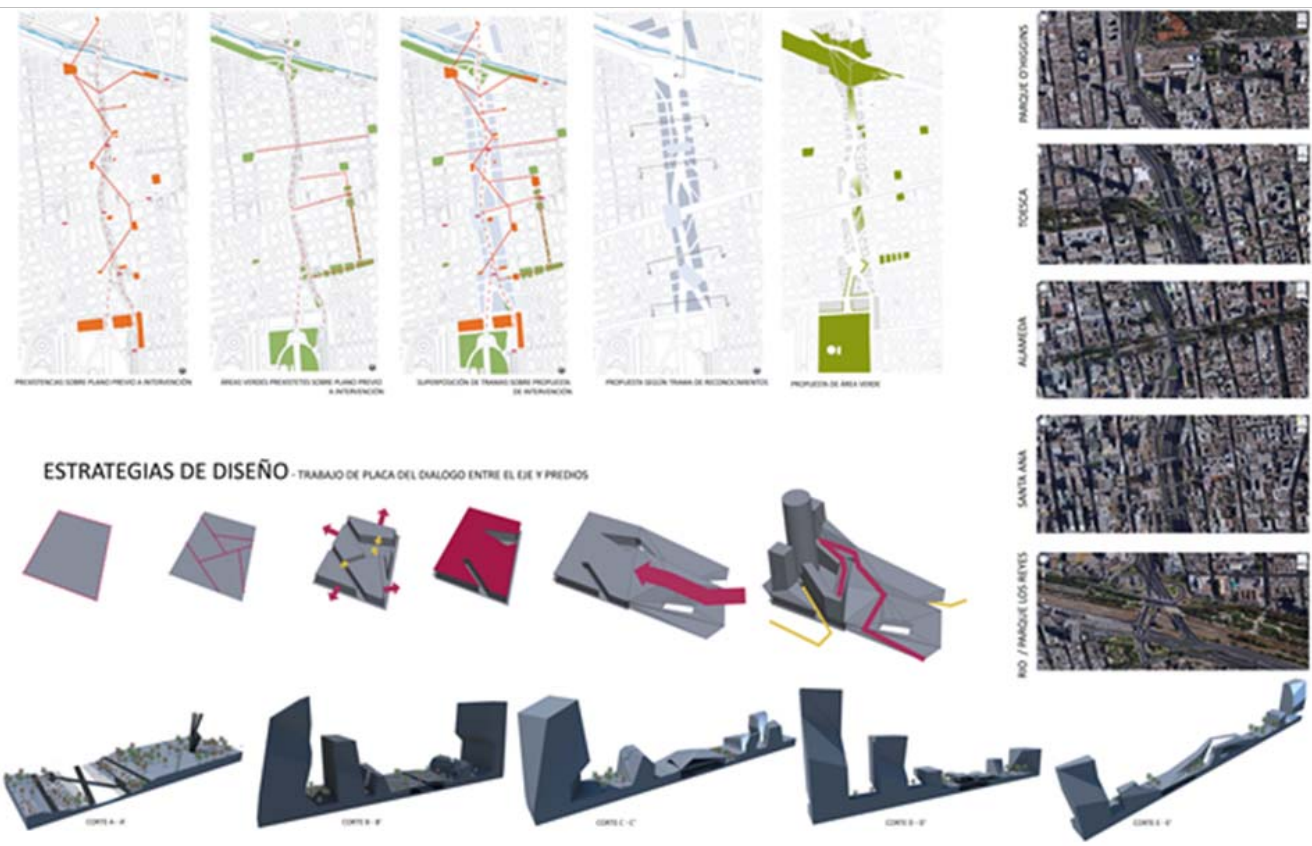

Fig 4. Propuesta intervención urbana Eje Norte-Sur. Fte.: Max Fernández, Daniela Moder, Diego Ponce.

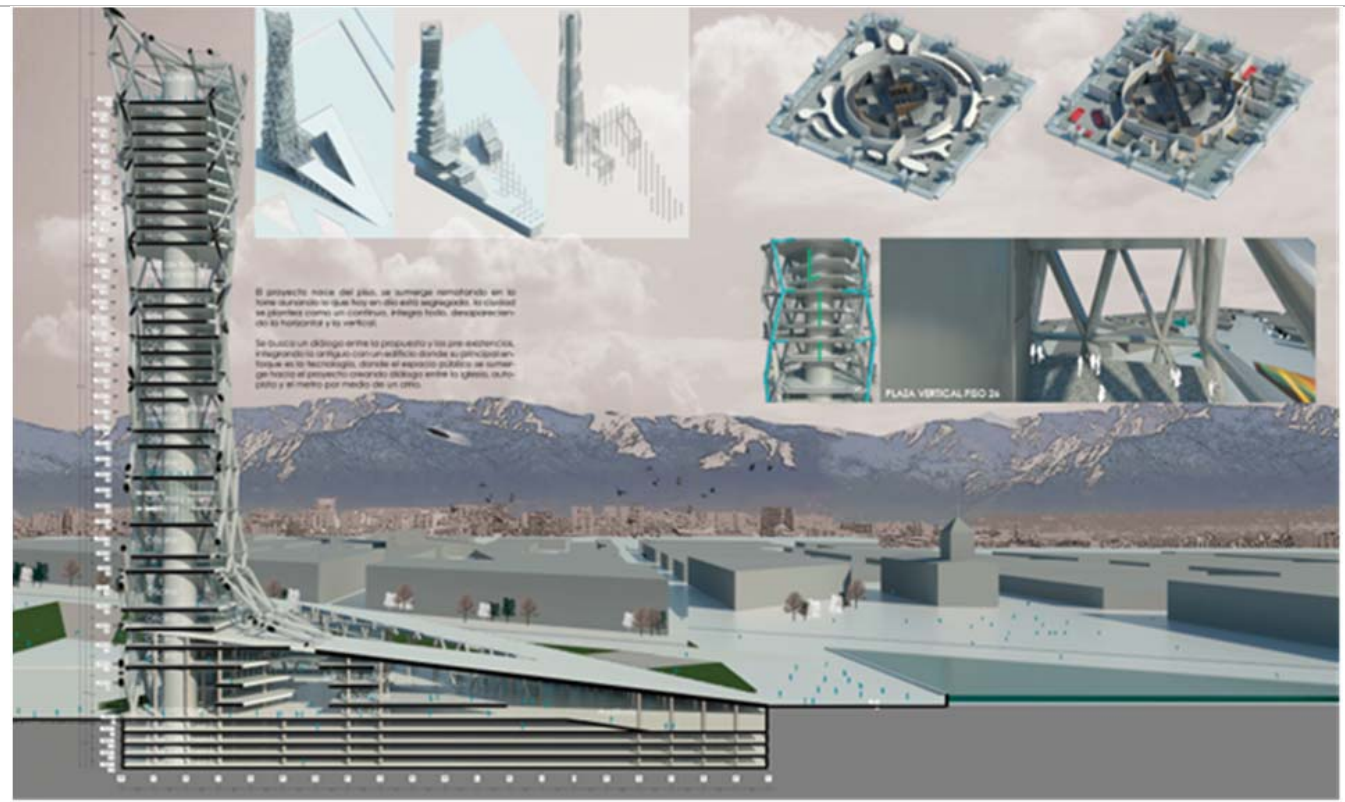

Fig 5. Propuesta individual. Centro Digital intervención urbana Eje Norte-Sur. Fte.: Max Fernández

12 


\section{Referencias Bibliográficas}

ALBERTI, L. Battista. (1991).De Re Aedificatoria. Prólogo de Javier Rivera; traducción de Javier Fresnillo Núñez. Madrid, España: Edición Akal. Originalmente publicado el año 1452.

ACSELRAD, H. (1999). Sustentabilidad y ciudad [versión electrónica]. Eure, 25 (74), 35-46

ALLEN, Stan. (1997). From objects to field. AD Profile 127 (Architecture after Geometry) Architectural Design vol.67 no.5/6 . Mayo-June 1997, p.24-31. Londres, Inglaterra: Academy Group Ltda.

ALLEN, Stan. (2000). Practice architecture, technique and representation. Australia: G+B Arts.

CORSINI, José María. (2004). Diseño urbano y pensamiento contemporáneo. Barcelona, España: Instituto Monsa de Ediciones, 2004.

DUANY, Andrés; Plater-Zyberk, Elizabeth. (1991). Towns And Town-Making Principles. Published in the United States of America by Rizzoli International Publications, Inc., New York, 1991. Edited by Alex Krieger with William Lennertz.

KATZ, Peter. (1994). The New Urbanism, Toward An Architecture Of Community. Afterword by Scully, Vincent. Mc Graw-Hill, Inc., 1994. Printed and bound in Hong Kong through Print Vision, Portland, Oregon.

LÓPEZ C., José. (1999). Diseño Urbano: Teoría y Práctica. Editorial Munilla - Leria, Madrid, España._Mabardi,Francois (Dir.). (2000). Taller en la enseñanza de la arquitectura. Concepción, Chile: Ediciones Universidad del Bío-Bío.

MABARDI, J.F. (2012). Maestría el proyecto. Apuntes para la práctica de la enseñanza del proyecto. Ediciones Universidad del Bío-Bío.

MUNIZAGA, Gustavo, (1992). Diseño urbano: teoría y método. Santiago, Chile: Ediciones Universidad Católica de Chile.

POZUETA E., Julio (Dir), LAMIQUIZ D., Francisco José, PORTO S., Mateus (2009). La Ciudad Paseable. Impresión Artegraf S.A., Madrid, 2009.

RICHARDSON, Harry W., BAE, Chang-Hee Christine (2004). Urban Sprawl in Western Europe and the United States. Aldershot, Reino Unido. 2004. 325 p. ISBN 10: 0754637891. ISBN-13: 978-0754637899. 